\title{
BLOOD OUTGROWTH ENDOTHELIAL CELL MIGRATION AND TRAPPING IN VIVO: A WINDOW INTO GENE THERAPY
}

\author{
L.C. Milbauer ${ }^{\star}$, J. Enenstein ${ }^{\star}$, M. Roney ${ }^{\star}$, A. Solovey ${ }^{\star}$, V. Bodempudi ${ }^{\star}$, T.C. Nichols ${ }^{\dagger}$, and \\ R.P. Hebbel ${ }^{\star}$ \\ * Vascular Biology Center and Division of Hematology-Oncology-Transplantation, Department of \\ Medicine, University of Minnesota Medical School, Minneapolis, MN, USA. \\ † Department of Medicine and Pathology, Francis Owen Blood Research Laboratory, University of \\ North Carolina, Chapel Hill, NC, USA.
}

\begin{abstract}
Human blood outgrowth endothelial cells (hBOEC) may be useful delivery-cells for gene therapy. hBOEC have high expansion capacity and stable phenotype. If incorporated into blood vessels, hBOEC could release therapeutic agents directly into the blood stream. However, little is known about lodging and homing of hBOEC in vivo. We examined the homing patterns of hBOEC in mice, and explored extending cell-based FVIII gene therapy from mice to larger animals. hBOEC were injected into NOD/SCID mice to determine where they localize, how localization changes over time and if there were toxic effects on host organs. The presence of hBOEC in mouse organs was determined by qPCR and immunofluorescence microscopy. hBOEC lodged most notably in mouse lungs at $3 \mathrm{~h}$, but by $24 \mathrm{~h}$ there were no differences between 9 organs. hBOEC longevity was assessed up to 7 months in vivo. hBOEC expanded well and then plateaued in vivo. hBOEC from older cultures expanded equally well in vivo as younger. hBOEC caused no noticeable organ toxicity up to 3 days post-injection. When mice were pretreated with antibodies to E-selectin, Pselectin or anti- $\alpha 4$ integrin prior to hBOEC injection, the number of hBOEC in lungs at $3 \mathrm{~h}$ was inhibited. Preliminary studies infusing hemophilic dogs with autologous canine BOEC overexpressing FVIII (B-domain deleted) showed improvement in whole blood clotting times (WBCT). In conclusion, the survivability, expandability and lack of toxicity of BOEC in vivo indicate that they may be valuable host cells for gene therapy.
\end{abstract}

\section{INTRODUCTION}

\begin{abstract}
Blood outgrowth endothelial cells (BOEC) are an attractive source of cells for repairing vascular injuries (i.e. post- infarction), seeding of vessel grafts, and as carrier cells in gene therapy. BOEC are mature endothelial cells that have been expanded from circulating progenitor cells from adult blood (1). Their high expansion capacity in culture makes BOEC well suited for auto-transplantation. In addition, their phenotype is quite stable. We previously showed that human BOEC (hBOEC) transfected with factor VIII (FVIII) gene released FVIII in vivo in mice over many months (2).
\end{abstract}

(C) 2008 Mosby, Inc. All rights reserved.

Corresponding author: Dr. Robert P. Hebbel, Vascular Biology Center and Divisions of Hematology-Oncology-Transplantation, Department of Medicine, University of Minnesota Medical School, Mayo Mail Code 480, 420 Delaware St. SE, Minneapolis, MN 55455. phone: 612-624-6104, FAX 612-625-6919, hebbe001@umn.edu .

Publisher's Disclaimer: This is a PDF file of an unedited manuscript that has been accepted for publication. As a service to our customers we are providing this early version of the manuscript. The manuscript will undergo copyediting, typesetting, and review of the resulting proof before it is published in its final citable form. Please note that during the production process errors may be discovered which could affect the content, and all legal disclaimers that apply to the journal pertain. 
In order to exploit the therapeutic potential of endothelial cells, it is necessary to understand their natural history after injection: their lodging, organ distribution, migration and expansion. Where cells lodge influences their efficacy as gene therapy agents since some vascular beds provide better microenvironments for long term maintenance and proliferation. Lodging is dependent both on physical factors (vessel structure, density, and flow rate) and on differential expression of cell surface adhesion molecules. Chemo-tactic agents may alter a cell's final location.

Very few studies have looked at which cell surface molecules mediate homing of endothelial cells or their progenitors to specific organs. Indeed, it is unclear whether endothelial cells home to specific organs or simply land and expand in more conducive environments. Our preliminary studies suggested that hBOEC injected in NOD/SCID mice localized primarily in bone marrow and spleen (2). In the present study we focused on the distribution of hBOEC over time in 9 organs, with special emphasis on lung, bone marrow and spleen. We evaluated the effect of cell surface molecules (both on hBOEC and mouse vessel beds) on hBOEC localization. hBOEC express variable amounts of the adhesion molecules: Vascular cell adhesion molecule (VCAM) and E-selectin, but negligible amounts of P-selectin, alpha-4 integrin and P-selectin glycoprotein ligand (PSGL1). The vascular beds of bone marrow, spleen and lungs differentially express VCAM, E-selectin and P-selectin. All three molecules are constitutively expressed in bone marrow (3)(4). Lung expresses all three molecules in large vessels (5). Alpha-4 integrin has been detected on proliferating but not quiescent endothelium (6). Histamine and LPS induce different organ specific effects on Eselectin and P-selectin (7).

This paper provides preliminary data on BOEC growth characteristics and the molecules which mediate their expansion in vivo. In addition, it extends our knowledge of cell-based FVIII gene therapy from mice to larger animals.

\section{MATERIALS AND METHODS}

Mice

NOD/SCID mice (Jackson labs), bred in our SPF facilities, were 8-12 wk old at the time of injection. All studies were done with the approval of the Institutional Animal Care and Use Committee at the University of Minnesota.

\section{hBOEC isolation and injection}

Blood for hBOEC isolation was obtained from healthy donors (age 19-30) according to ethical protocols at the University of Minnesota. hBOEC were isolated as described in (1). hBOEC pooled from frozen and passed stocks of 4 donors (passage 5 or 6 ) were used for tail vein injection. Each injection contained $4 \times 10^{5} \mathrm{hBOEC}$ in $0.1 \mathrm{ml}$ mouse saline or $8 \times 10^{5}$ hBOEC in $0.2 \mathrm{ml}$ mouse saline. FACS analysis (1), of all experiments, ensured the endothelial character of the hBOEC. Positive markers for endothelial cells included VE cadherin and P1H12. The negative marker was CD14.

\section{及2-microglobulin/PECAM (CD31) staining to detect human BOEC in mouse tissue}

Frozen sections $(5 \mu)$ were acetone fixed 10 minutes and stored at $-80^{\circ} \mathrm{C}$. For use, sections were fixed with $4 \%$ PFA/PBS; blocked with $3 \%$ BSA/PBS; stained $1 \mathrm{~h}$ with rabbit antihuman $\beta 2$-microglobulin (1:200) (Accurate Chemicals) and rat anti-mouse CD31 (1:100) (BD BiosciencesPharmingen); treated with secondary donkey anti-rabbit/TRITC (Jackson Labs) and anti-rat /FITC for 30 min and counterstained with DAPI. 


\section{Toxicity analysis}

Paraffinized sections $(5 \mu)$ or frozen sections were stained with hematoxilin/eosin and viewed for tissue integrity.

\section{Mouse Blocking Experiments}

NOD/SCID mice were tail-vein injected with 50 micrograms of azide-free rat anti-mouse antibodies from BD Pharmingen: anti- $\alpha 4$ integrin IgG2a (clone R1-2), anti P-selectin IgG1 (clone RB40.34), anti E-selectin IgG2a (clone 10E 9.6), anti-VCAM IgG2a (clone 429), anti-PSGL1 IgG1 (clone 2PH1), and saline, or normal rat IgG (Southern Biotech) $1 \mathrm{~h}$ prior to injection of $8 \times 10^{5} \mathrm{BOEC} /$ mouse.

\section{BOEC blocking experiments}

$8 \times 10^{5} \mathrm{hBOEC} /$ mouse were pretreated with 10 micrograms of azide-free mouse anti-human antibody for 30 minutes and rinsed prior to tail vein injection. Mouse anti-human antibodies included: anti- $\alpha 4$ integrin IgG1 (BU49) (Calbiochem), anti-P-Selectin IgG1 (Clone AK4, BD Pharmingen), anti-VCAM IgG1 (HAE-2Z, Santa Cruz), or anti-VCAM IgG1(1G11, Beckman), anti-PSGL1 (3E2.25.5, Beckman), anti-E-selectin IgG1 (Hae-1f, Ancell) and normal mouse IgG (Southern Biotech).

\section{Development and analysis of canine FVIII-expression in canine BOEC (CBOEC)}

cBOEC from three Hemophilia A dogs (E64, D28 and H17, Chapel Hill HA colony, NC) (8) were isolated according to protocols (1). cBOEC expressed normal endothelial markers: PECAM, von Willebrand factor (vWF) and LDL. All procedures were approved by the University of North Carolina Institutional Animal Care and Use Committee.

cBOEC were transduced with retroviral particles containing MIRG/cFVIII (constructed from pBK-CMV-FVIIIcDNAbdd; a gift from Dr. Lillicrap, Queen's University, Canada). Positive cFVIII producing cBOEC (cBOEC/FVIII) (named tdH64, $\mathrm{tdH} 28$ and tdH17) were selected by sterile FACS sorting. cFVIII concentration and activity produced by $\mathrm{cBOEC} /$ FVIII were detected by IMUBIND FVIII ELISA kit (American Diagnostic) and Coamatic Factor VIII Kit (Chromogenix) respectively. cBOEC/FVIII expressed endothelial markers and took up LDL. The copy number of transgene in $\mathrm{cBOEC/cFVIII} \mathrm{was} \mathrm{examined} \mathrm{by} \mathrm{real-}$ time qPCR.

\section{Infusion}

Three hemophilia A dogs (E64, D28 and H17) in Chapel Hill HA colony were infused with autologous cBOEC/FVIII. Dog ages at the start of infusions were E64 (3.6 years), D28 (5.6 years), and $\mathrm{H} 17$ (1.8 years). For each infusion, 65 to 136 million viable autologous cBOEC/ FVIII cells per $150 \mathrm{ml}$ of saline were infused into each hemophilia A dog through the cephalic vein. Blood samples were collected periodically for monitoring WBCT, hematological parameters and liver and kidney function.

\section{Whole Blood Clotting Assay}

WBCT were performed by a two-tube procedure at $28^{\circ} \mathrm{C}$. One $\mathrm{ml}$ of whole blood was collected and distributed equally into two siliconized tubes (Becton-Dickinson). The first tube was tilted every $30 \mathrm{sec}$. After a clot formed, the second tube was tilted and observed every $30 \mathrm{sec}$. The endpoint was the clotting time of the second tube. 


\section{Real-time DNA Quantitative PCR}

Genomic DNA from BOEC and from each animal organ was isolated immediately using DNeasy Tissue Kit (Qiagen) according to the manufacturer's protocol. Genomic DNA realtime qPCR was performed using I Cycler (Bio-Rad) for the quantitation of hBOEC in each mouse organ. Primers and 3' labeled fluorescence FAM-probe for human $\beta 2$-microglobulin were designed to amplify human genomic DNA specifically. Sequences are as follow: sense primer -5'-GCT GGA TTG GTA TCT GAG GCT AG-3'; antisense primer-5'- GCT GTT CCT ACC CAT GAA TAC AT-3'; 5'-FAM-AAG GGC TTG TTC CTG CTG GGT AGC TCT AAA C-TAMRA-3'. The kit of TaqMan Rodent GAPDH Control Reagents that contains universal primers and fluorescence VIC- probe (Applied Systems) were used for internal control for amplifying both human and mouse genomic DNA. Standard curves were set up by serially diluting human genomic DNA in mouse genomic DNA. Each real-time PCR reaction contained 500ng of genomic DNA, 200nM of each primer, $150 \mathrm{nM}$ of each probe and $1 \times$ of iQ supermix (Bio-Rad Laboratories). The PCR condition was as follows: Denaturation at $95^{\circ} \mathrm{C}$ for 5 minutes following by 60 cycles of two-step PCR $\left(95^{\circ} \mathrm{C}\right.$ for 30 seconds and $60^{\circ} \mathrm{C}$ for 30 seconds). Modified Ct (Threshold Cycle) value was calculated by dividing the $\mathrm{Ct}$ of hB2M with that of GAPDH. Percent of human cells in each mouse organ was calculated based on the standard curve. Sequences of probes and primers for the copy number of transgene in $\mathrm{cBOEC} / \mathrm{cFVIII}$ and the quantity of cBOEC/cFVIII in canine organs were as follow: Sense-5' -CAT CGG CGT GCA GTG CTT-3'; Antisense-GGG CAT GGC GGA CTT G-3'; Probe-5'FAM-CCG CTA CCA CAT GAA GC-TAMRA-3'. Serial dilution of cBOEC/FVIII genomic DNA in cBOEC genomic DNA was used as a standard curve.

\section{Statistics}

Two-way Analysis of Variance (ANOVA) model was used to investigate effects of time periods and organ types on percentage of hBOEC in mice. All multiple comparison p-values were adjusted by the Tukey-Kramer procedure (9), and adjusted p-values $<0.05$ were ascertained to be statistically significant. All statistical analyses were performed using SAS 9.0 (9). Since the effect of time periods on percentage of hBOEC differed between different organs ( $\mathrm{F}$ test $\mathrm{p}$-value $=.015$ ), we additionally fit separate one-way ANOVA models for each organ to investigate organ-specific time effects.

\section{RESULTS}

Understanding the natural history of hBOEC in their NOD/SCID hosts is an important antecedent to properly designing gene therapy studies. We must know which organs are amenable to initial seeding, whether the same organs are conducive to hBOEC expansion, and whether hBOEC growth plateaus.

We concentrated on 4 questions. 1) What is the relative distribution of hBOEC in 9 organs at $3 \mathrm{~h}$ and $24 \mathrm{~h}$ post injection? 2) Are toxic effects present at early time points? 3) How much do hBOEC expand in various organs at 2 wks, 2 months and 4 months? 4) Does the age of $\mathrm{hBOEC}$ at the time of injection affect expansion capacity?

hBOEC were visible in all tested organs (brain, gut, heart, kidney, liver, lung, ovaries/testes, spleen and bone marrow) by their positive staining for human $\beta 2$-microglobulin and characteristic large size. Transplanted cells were present as single cells or trains of cells, not clumps, in all tissue samples. A number of large $\beta 2$-microglobulin positive hBOEC were noted in capillaries, and larger vessels, but rarely in alveoli and bronchi in lungs of all animals after $3 \mathrm{~h}$ and much fewer at $24 \mathrm{~h}$ and 3 days (Fig1). Stained cells were found mostly but not exclusively in peribronchial areas. Numbers of cells varied greatly from animal to animal and from section to section. 
The amount of hBOEC in 9 mouse organs at $3 \mathrm{~h}$ and $24 \mathrm{~h}$ post tail vein injection was determined by real-time quantitative polymerase chain reaction (qPCR) of hBOEC per total cells per organ (Fig 2). Data were analyzed by ANOVA and t-test. $3 \mathrm{~h}$ post-injection, lung had significantly higher percentage of hBOEC than any other organs (all Tukey-Kramer adjusted $\mathrm{p}$-values $<0.0001$ ), whereas at $24 \mathrm{~h}$ there was no significant difference between organs. Percentage of hBOEC decreased significantly from $3 \mathrm{~h}$ to $24 \mathrm{~h}$ in gut, heart, liver, lung, spleen and bone marrow (all t-test p-values $<0.05$ ), and decreased with marginal significance in kidney and testis. The decrease in hBOEC in most organs at $24 \mathrm{~h}$ suggests that $\mathrm{hBOEC}$ are either dislodged, or die and are cleared.

Two sets of tissues were examined for toxicity: $3 \mathrm{~h}$ and $24 \mathrm{~h}$ (frozen sections), and $3 \mathrm{~h}$ and 3 days (paraffinized sections) (8 mice per group). No significant difference in morphology was observed between animals injected with hBOEC versus control animals that received only saline at $3 \mathrm{~h}, 24 \mathrm{~h}$ and $3 \mathrm{~d}$. In both experimental groups, severe lymphocyte depletion was noticed in all animals (this is characteristic for NOD/SCID mice). Dr. Carlson (veterinary diagnostic lab, University of Minnesota) independently confirmed that there was no significant difference between the control group and hBOEC recipients. These results extend our preliminary studies at $9 \mathrm{~d}, 28 \mathrm{~d}$ and $156 \mathrm{~d}$ (2). No thrombosis, occlusions or infarcts were evident in either that preliminary study or the present study.

Expansion of hBOEC in 9 different organs was monitored at 2 wks, 2 months, and 4 months (Fig 3). Expansion capacity was determined from percent of hBOEC/total cells/organ at different time points. ANOVA was used to analyze overall expansion, as well as time and organ effects for individual organs. Averaged over organs, percentage of hBOEC significantly decreased from 2 wks to 2 months ( $\mathrm{p}=.003$ ), while significant increase was seen at 4 months compared to both 2 wks and 2 months ( $\mathrm{p}=.001$ ). For all organs but heart and kidney, there was significant increase in percentage of human cells from 2 wks to 4 months. In addition, all organs except spleen, had significantly increased percentage of human cell from 2 months to 4 months.

Average increase from two wks to four months was 6-fold for bone marrow and spleen, 3fold for liver and testis/ovary, and 1.5-2-fold for other organs. This indicates that many organs provide a suitable environment for hBOEC expansion. Brain, kidney and liver showed significant decrease from 2 wks to 2 months. The drop in hBOEC between 2 wks and 2 months might indicate cell death followed by bursts of expansion of remaining hBOEC. Another experiment monitored expansion of hBOEC in bone marrow and spleen out to 7 months. hBOEC levels plateaued between months 4 and 5 (not shown). There was no significant difference in mortality rate between injected and uninjected mice at 7 months.

Conceivably, the age of hBOEC at the time of injection might affect their ability to expand and survive. hBOEC were tail vein injected at passage 5, 10 and 15, and analyzed 6 months later in bone marrow, spleen and lung (Fig 4). There was no significant difference in vivo between earlier and later passages of hBOEC. hBOEC from passage 15 grew and survived in vivo as well as passages 5 or 10 from the same donor. FACS analysis showed that VCAM and VE-cadherin expression remained stable through passage 10, but VE-cadherin expression declined in some donors by passage 15 (not shown).

Many hBOEC initially lodge in lungs at three hours. We wanted to know which cell adhesion molecules might be involved in lodging and whether we could decrease lodging in lungs and simultaneously increase lodging in other organs, such as bone marrow or spleen, by pre-treating either the mice or hBOEC with antibodies to cell adhesion molecules.

Mice were pretreated with antibodies to E-selectin, P-selectin or $\alpha 4$ integrin for $1 \mathrm{~h}$ prior to tail vein injection of hBOEC (two experiments). Organs were harvested at 3-4 $\mathrm{h}$ from lung, 
bone marrow, and spleen (Fig 5). Anti-E-selectin, anti-P-selectin or anti- $\alpha 4$ integrin antibodies inhibited mouse tissue ( $\mathrm{P}<.01$ versus controls), so that fewer hBOEC lodged in the lungs (Fig 5a). Bone marrow lodging was not significantly inhibited with anti- $\alpha 4$ integrin (Fig 5b). Studies were inconclusive for P- or E-selectin in bone marrow and for all 3 antibodies in spleen. Only 1 set of experiments had mice pretreated with anti-VCAM or anti PSGL-1 (not shown). Anti-VCAM did not inhibit lodging in lung, bone marrow or spleen. PSGL-1 blocked lodging in spleen $\mathrm{P}<0.01$ versus either saline or control antibody and lung (P-value 0.01 versus saline, but not control antibody).

FACS analysis of unstimulated hBOEC showed that they express variable amounts of surface VCAM $(32 \%+/-27 \%)$ and E-selectin $(11 \%+/-15 \%)$, and less than $10 \% \mathrm{P}-$ selectin, $\alpha 4$-integrin and PSGL1-1. 90\% of TNF $\alpha$ stimulated hBOEC expressed VCAM. These results suggested that anti-VCAM treatment might be the most effective hBOEC inhibitor, but this would depend on the activation state of the hBOEC. When unstimulated hBOEC were pretreated with antibodies to VCAM, $\alpha 4$-integrin or P-selectin for 30 minutes prior to tail vein injection, there was no significant inhibition of homing to lung, bone marrow or spleen (not shown).

These results suggest that E-selectin, P-selectin and $\alpha 4$ integrin on lung vasculature may be involved in hBOEC lodging. Since inhibition was partial, it is likely that mechanical forces are also involved in lodging. It is also likely that lung $\alpha 4$ integrin is binding to some other ligand than VCAM, since anti-VCAM pretreatment of hBOEC did not inhibit lodging.

The canine model of hemophilia has been widely used for developing and evaluating gene therapies (10). We chose the canine model to evaluate the use of canine BOEC (cBOEC) as an autologous cell-based gene therapy for hemophilia A. cBOEC were isolated from three Hemophilia A dogs. The cBOEC were manipulated to over-express canine FVIII. Canine FVIII producing cBOEC lines (tdE64, tdD28 and tdH17) showed standard endothelial markers and LDL uptake that were the same as those of their parental cBOEC (data not shown). FVIII production from 1 million cells in $10 \mathrm{ml}$ culture medium of cBOEC/FVIII was $\sim 250 \mathrm{ng} / \mathrm{ml}$. Transgene copy number per cell of cBOEC/FVIII was 4.6-5.3.

Autologous cBOEC/FVIII cells were infused into three hemophilia A dogs. Whole blood clotting times (WBCT) in the three dogs were clearly improved after infusions (Fig. 6). Whereas baseline WBCT was $>60$ minutes in each of these dogs, post infusion WBCT repeatedly dipped below 40 minutes, and sometimes below 30 minutes. (The historical range for hemophilia A dogs is 40 to $>60$ minutes). After the initial drop in WBCT at 2-14 days, the WBCT rose in all 3 dogs, before dropping again. This may indicate that infused cBOEC/ FVIII cell numbers (and FVIII expression) decline before rebounding. Although this improvement did not meet the normal range (8 to 12 minutes), it is important to note that the improvement in WBCT lasted many months. As a control, we infused non-transfected canine BOEC into one hemophilia A dog. WBCT in the control did not decrease at $1 \mathrm{hr}, 4 \mathrm{hr}$ or $24 \mathrm{hr}$ post infusion (not shown). Also noteworthy is the dearth of bleeding incidents in these BOEC/FVIII treated dogs (1 per year or less), whereas hemophilia A dogs have, on average, six bleeding incidents per year. Cell infusions had no dramatic effect on either the animals' hematological parameters or liver and kidney functions (not shown). However, infusions tended to be associated with mild transient thrombocytopenia.

Distribution of autologous cBOEC/FVIII in 11 organs was examined by qPCR in 2 dogs: dog E64 (430d after first infusion) and dog H17 (160d after first infusion). Most of the cBOEC/FVIII homed to liver in both dogs $(0.2 \%$ and $0.4 \%$ of liver cells respectively) after the infusions, with heart retaining the second highest levels $(0.02 \%$ and $0.155 \%$ respectively). Further studies are needed to corroborate these preliminary findings. 


\section{DISCUSSION}

We have shown that hBOEC lodge in many mouse organs, most notably lungs, within $3 \mathrm{~h}$ of tail vein injection, and that the number of $\mathrm{hBOEC}$ in lungs and other organs decreases significantly by $24 \mathrm{~h}$. There were no significant signs of toxicity in the hosts at $3 \mathrm{~h}, 24 \mathrm{~h}$ or 3 days post injection. hBOEC expanded in all 9 organs examined over 4 months. hBOEC populations reached plateaus in host organs. hBOEC from older cell culture (passage 15) were equally able as younger hBOEC to expand in vivo. The survivability, expandability and lack of toxicity of hBOEC indicate that they are a useful tool for gene therapy.

In mice, bone marrow and spleen consistently displayed high expansion capacity for hBOEC. By contrast, hBOEC initially lodged in lung in much higher numbers than in bone marrow or spleen but lung had lower expansion capacity from 2 wks to 4 month. The number of hBOEC can be the result of lodging, cell division, migration, or death. A recent study of porcine BOEC $24 \mathrm{~h}$ post injection also showed a wide distribution in mouse tissues. The highest concentrations were in liver and lung (11).

The number of hBOEC detected in the nine different organs was a small fraction of the total injected. From the literature it appears that BOEC or progenitor cell lodging is normally low in healthy organs (12) (13). Recipient NK cells or macrophages may deplete the population (14). E-selectin on bone marrow may promote apoptosis of mobilized CD34 ${ }^{+}$cells (3).

We began studies on cell surface molecules that might be involved in early lodging and homing of hBOEC. We focused on molecules, such as $\alpha 4$ integrin, E-selectin, P-selectin, VCAM and PSGL-1 that have been implicated in homing of stem cells or EPC's. Pretreatment of mice with antibodies to E-selectin, P-selectin, or $\alpha 4$ integrin inhibited lodging of hBOEC in the lung at $3 \mathrm{~h}$. Since inhibition was only partial, future studies should determine whether other molecules affect lodging and which ligands on hBOEC bind to lung endothelium. Known ligands for $\alpha 4 / \beta 1$ integrin include VCAM, the HepII/IIICS domain of fibronectin (15), and propolypeptide of vWF (16). Ligand binding of $\alpha 4$ integrin is complex and therefore may be difficult to detect and inhibit in vivo. $\alpha 4$ integrin is expressed on proliferating but not quiescent endothelial cells (6) and exists in both low affinity and high affinity states (17).

Homing studies with hematopoietic progenitor cells envision $\alpha 4$ integrin on these cells and E-or P-selectin or VCAM on host endothelium (18). By contrast, we suggest that $\alpha 4$ integrin on host lung endothelium binds to some ligand on hBOEC. Since pretreatment of hBOEC with antibodies to VCAM, had no effect on lodging of hBOEC in lungs, it is possible that either the HepII/IIICS domain of fibronectin or VWF is forming a bridge between $\alpha 4$ integrin on lung endothelium and hBOEC.

The cell surface molecules that control BOEC lodging in uninjured vasculature may differ from homing to injured vasculature. Hind limb ischemia experiments may provide insights into how BOEC might respond to activated blood vessels. Homing capacity of endothelial cells and EPC's to ischemic hind limb was altered by several antibodies. Yang (19) expanded human $\mathrm{Cd} 133^{+}$cord blood cells into differentiated endothelial cells and tail vein injected these into nude mice, where they incorporated into blood vessels in ischemic hindlimb. Incorporation was decreased by pretreating endothelial cells with anti-PSGL-1. Duan (20) found that VCAM was upregulated in vessel endothelium of ischemic mouse tissue. Recovery of hind limb blood flow with EPC's was inhibited when these cells were pretreated with antibodies to $\alpha 4$ integrin.

Our preliminary studies of autologous cBOEC/FVIII infused into hemophilia A dogs resulted in modest decreases in clotting times that were sustained up to 4 months. Although 
these decreases never reached normal levels, improvements of the method are readily envisioned. Dogs were infused with $\sim 1 / 10$ the BOEC number $/ \mathrm{kg}$ of host as in the mice. Increasing cBOEC/FVIII number10-fold in dogs would probably increase the amount of FVIII delivered, thus improving clotting time. Treatment might also be improved with young puppies since host size would be smaller, thus decreasing the number of $\mathrm{cBOEC} /$ FVIII needed; and the natural growth of pup blood vessels might provide more in vivo sites for cBOEC/FVIII incorporation and expansion. Modifications in the promoter region of the FVIII expression vector could improve efficiency of expression, and modifications in the $3^{\prime}$ untranslated region could increase RNA stability. Modifications of FVIII at charged amino acid residues could increase protein stability (21).

Although there has been some success with viral vector infusion therapy in hemophilia (22), BOEC based gene therapy offers several advantages. Since the FVIII gene is only introduced ex vivo, the chance of protein synthesis of FVIII increases, whereas the chance of unexpected effects decreases; no virus is introduced into other cell types in vivo. Unexpected effects might be further diminished by implanting FVIII expressing BOEC subcutaneously in a Matrigel ${ }^{\mathrm{TM}}$ scaffold (23). BOEC also have an advantage over hematopoietic stem cell based gene therapy because BOEC will not differentiate into a wide variety of cell types with unpredictable outcomes.

BOEC are a potential resource for repopulating wounded endothelium and a potential host cell for gene therapy. Endothelial cells are particularly valuable as hosts because they could release the therapeutic gene product directly into the blood stream. These in vivo studies provide further evidence that BOEC have growth characteristics and lack of toxicity that would make them suitable as carrier cells for gene therapy.

\section{Acknowledgments}

We thank Dr. Cathy S. Carlson (University of Minnesota) for corroborative toxicity analysis of tissue samples. We thank Fuad Abdulla, Matt Hass, Darrell Johnson, James Kiley, Rahn Kollander and Julia Nguyen for technical assistance, and Peng Wei for statistical analysis.

This work has been sponsored by NIH grant \#HL71269, a National Hemophilia Foundation laboratory grant, and Resource Grant \#HL63098. The authors do not have any financial conflicts of interest.

\section{Abbreviations}

$\begin{array}{ll}\text { FVIII } & \text { factor eight } \\ \text { cBOEC } & \text { canine blood outgrowth endothelial cells } \\ \text { hBOEC } & \text { human blood outgrowth endothelial cells } \\ \text { PSGL1 } & \text { P-selectin glycoprotein ligand } \\ \text { qPCR } & \text { quantitative polymerase chain reaction } \\ \text { VCAM } & \text { vascular cell adhesion molecule } \\ \text { VWF } & \text { von Willebrand factor } \\ \text { WBCT } & \text { whole blood clotting time }\end{array}$

\section{REFERENCES}

1. Lin Y, Weisdorf D, Solovey A, Hebbel RP. Origins of circulating endothelial cells and endothelial outgrowth from blood. J Clin Invest. 2000; 105(1):71-77. [PubMed: 10619863] 
2. Lin Y, Chang L, Solovey A, Healey JF, Lollar P, Hebbel RP. Use of blood outgrowth endothelial cells for gene therapy for hemophilia A. Blood. 2002; 99(2):457-62. [PubMed: 11781225]

3. Winkler IG, Snapp KR, Simmons PJ, Lévesque JP. Adhesion to E-selectin promotes growth inhibition and apoptosis of human and murine hematopoietic progenitor cells independent of PSGL-1. Blood. 2004; 103(5):1685-92. [PubMed: 14592840]

4. Schweitzer KM, Dräger AM, van der Valk P, Thijsen SF, Zevenbergen A, Theijsmeijer AP, et al. Constitutive expression of E-selectin and vascular cell adhesion molecule-1 on endothelial cells of hematopoietic tissues. Am J Pathol. 1996; 148(1):165-75. [PubMed: 8546203]

5. Feuerhake F, Füchsl G, Bals R, Welsch U. Expression of inducible cell adhesion molecules in the normal human lung: immunohistochemical study of their distribution in pulmonary blood vessels. Histochem Cell Biol. 1998; 110(4):387-94. [PubMed: 9792417]

6. Garmy-Susini B, Jin H, Zhu Y, Sung RJ, Hwang R, Varner J. Integrin alpha4 beta1-VCAM-1mediated adhesion between endothelial and mural cells is required for blood vessel maturation. $\mathrm{J}$ Clin Invest. 2005; 115(6):1542-51. [PubMed: 15902308]

7. Eppihimer MJ, Wolitzky B, Anderson DC, Labow MA, Granger DN. Heterogeneity of expression of E- and P-selectins in vivo. Circ Res. 1996; S79(3):560-9. [PubMed: 8781489]

8. Lozier JN, Dutra A, Pak E, Zhou N, Zheng Z, Nichols TC, et al. The Chapel Hill hemophilia A dog colony exhibits a factor VIII gene inversion. Proc Natl Acad USA. 2002; 99(20):12991-6.

9. SAS 9.0 online documentation. http://support.sas.com/documentation/index.html

10. Casal M, Haskins M. Large animal models and gene therapy. Eur J Hum Genet. 2006; 14(3):26672. [PubMed: 16333317]

11. Smits PA, Kleppe LS, Witt TA, Mueske CS, Vile RG, Simari RD. Distribution of circulationderived endothelial progenitors following systemic delivery. Endothelium. 2007; 14(1):1-5. [PubMed: 17364891]

12. Asahara T, Murohara T, Sullivan A, Silver M, van der Zee R, Li T, et al. Isolation of putative progenitor endothelial cells for angiogenesis. Science. 1997; 275(5302):964-6. [PubMed: 9020076]

13. Wei J, Jarmy G, Genuneit J, Debatin KM, Beltinger C. Human blood late outgrowth endothelial cells for gene therapy of cancer: determinants of efficacy. Gene Therapy. 2007; 14(4):344-56. [PubMed: 17024106]

14. Yoshino H, Ueda T, Kawahata M, Kobayashi K, Ebihara Y, Manabe A, et al. Natural killer cell depletion by anti-asialo GM1 antiserum treatment enhances human hematopoietic stem cell engraftment in NOD/Shi-scid mice. Bone Marrow Transplant. 2000; 26(11):1211-6. [PubMed: 11149733]

15. Clements JM, Newham P, Shepherd M, Gilbert R, Dudgeon TJ, Needham LA, et al. Identification of a key integrin-binding sequence in VCAM-1 homologous to the LDV active site in fibronectin. J Cell Sci. 1994; 107(Pt 8):2127-35. [PubMed: 7527054]

16. Isobe T, Hisaoka T, Shimizu A, Okuno M, Aimoto S, Takada Y, et al. Propolypeptide of von Willebrand factor is a novel ligand for very late antigen-4 integrin. J Biol Chem. 1997; 272(13): 8447-53. [PubMed: 9079671]

17. Yednock TA, Cannon C, Vandevert C, Goldbach EG, Shaw G, Ellis DK, et al. Alpha 4 beta 1 integrin-dependent cell adhesion is regulated by a low affinity receptor pool that is conformationally responsive to ligand. J Biol Chem. 1995; 1270(48):28740-28750. [PubMed: 7499396]

18. Kopp HG, Avecilla ST, Hooper AT, Rafii S. The bone marrow vascular niche: home of HSC differentiation and mobilization. Physiology (Bethesda). 2005; 20:349-56. [PubMed: 16174874]

19. Yang C, Zhang ZH, Li ZJ, Yang RC, Qian GQ, Han ZC. Enhancement of neovascularization with cord blood CD133+ cell-derived endothelial progenitor cell transplantation. Thromb Haemost. 2004; 91(6):1202-12. [PubMed: 15175808]

20. Duan H, Cheng L, Sun X, Wu Y, Hu L, Wang J, et al. LFA-1 and VLA-4 involved in human high proliferative potential-endothelial progenitor cells homing to ischemic tissue. Thromb Haemost. 2006; 96(6):807-15. [PubMed: 17139377] 
21. Wakabayashi H, Varfaj F, Deangelis J, Fay PJ. Generation of enhanced stability factor VIII variants by replacement of charged residues at the A2 domain interface. Blood. Jul 23.2008 [Epub ahead of print].

22. Ponder KP. Gene therapy for hemophilia. Curr Opin Hematol. 2006; 13(5):301-7. [PubMed: 16888433]

23. Matsui H, Shibata M, Brown B, Labelle A, Hegadorn C, Andrews C, et al. Ex vivo gene therapy for hemophilia A that enhances safe delivery and sustained in vivo factor VIII expression from lentivirally engineered endothelial progenitors. Stem Cells. Oct; 2007 25(10):2660-9. [PubMed: $17615271]$ 

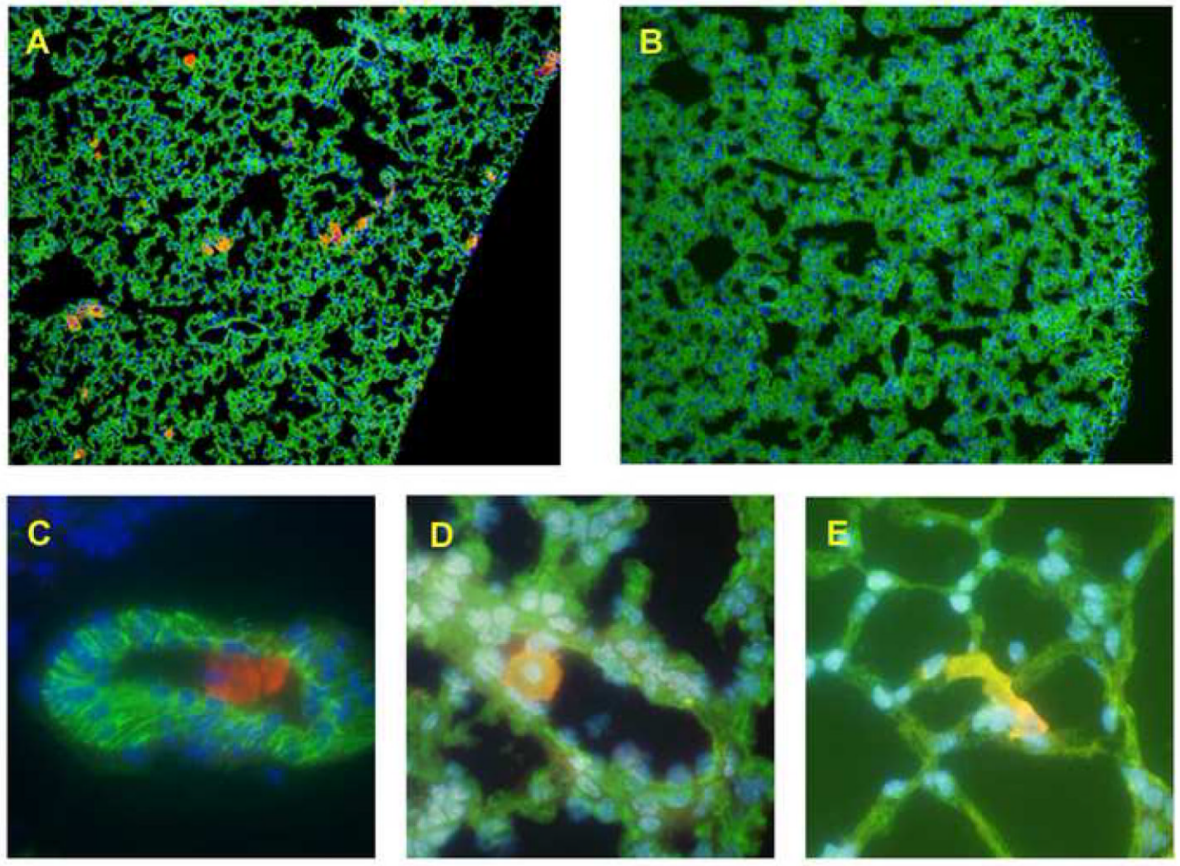

Fig 1. hBOEC localization in lung tissue

$\beta 2$-microglobulin positive $\mathrm{hBOEC}$ are present in lung tissue at $3 \mathrm{~h}$ post tail vein injection. (A) hBOEC injected mice, 15x magnification; (B) Artery, 60x magnification; (C) Vein, $60 \times$ magnification; (D) Capillaries, $60 \times$ magnification. (E) hBOEC within capillary, $60 \times$ magnification. $\beta 2$-microglobulin $=$ red; PECAM = green; $\beta 2$-microglobulin-PECAM overlap $=$ yellow. Dapi nuclei $=$ blue . 


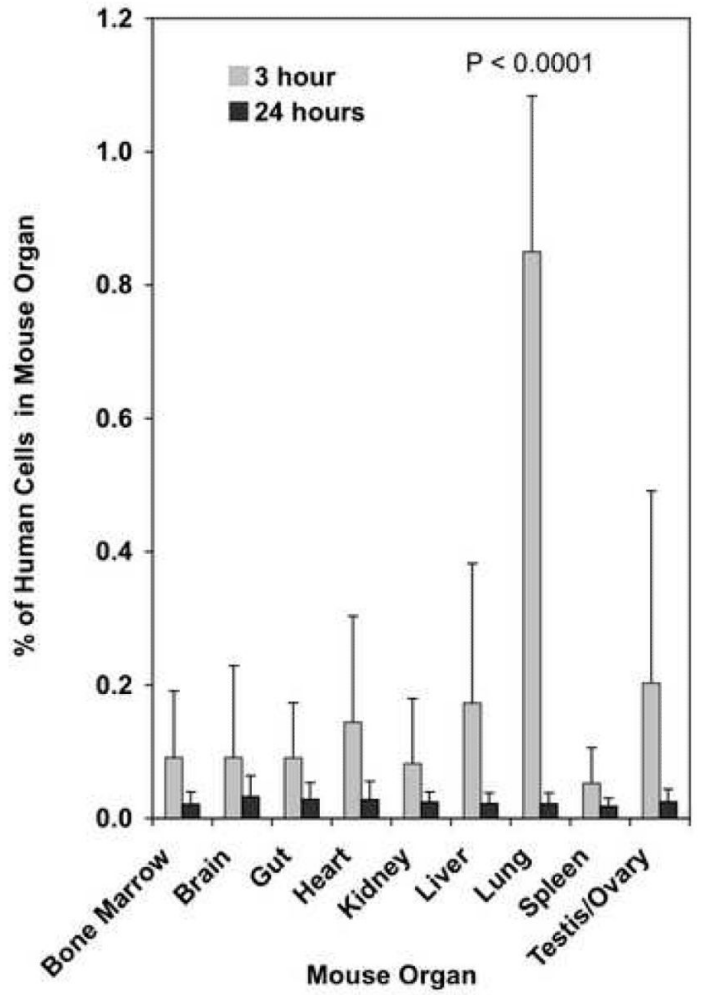

Fig 2. Distribution of total hBOEC in 9 mouse organs $4 \times 10^{5} \mathrm{hBOEC} / \mathrm{mouse}$ were tail-vein injected into NOD/SCID mice ( 8 mice per time point). Organs were harvested at $3 \mathrm{~h}$ and $24 \mathrm{~h}$ and analyzed by real-time qPCR. Data represents $\%$ hBOEC per total cells per organ. Error bars represent standard deviations. P-value is for lung versus other organs. One experiment. 


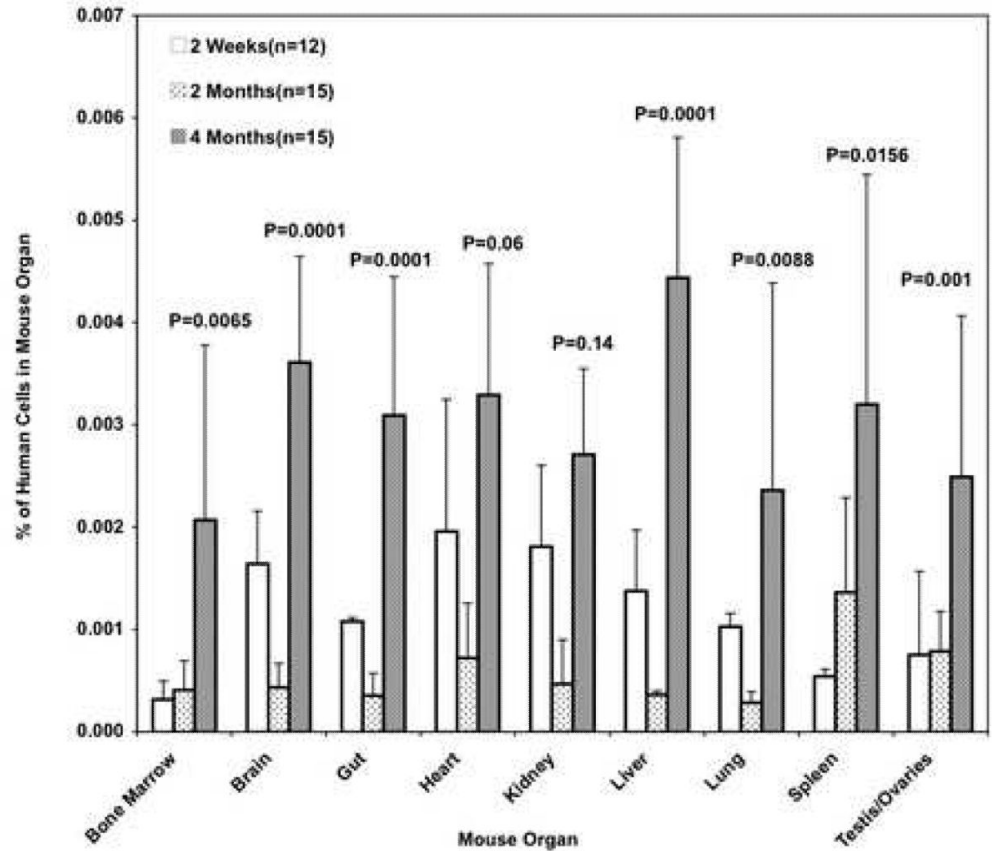

Fig 3. hBOEC expansion in 9 organs at 2 wks, 2 months and 4 months

$8 \times 10^{5} \mathrm{hBOEC} / \mathrm{mouse} /$ day were tail-vein injected into NOD/SCID mice on 3 consecutive days. Organs were harvested at 2 wks, 2 months, and 4 months and analyzed by real-time qPCR (12-15 mice per group). Data represents \% hBOEC per total cells per organ. Error bars represent S.D. P-values are for 2 wks versus 4 months. One experiment. 


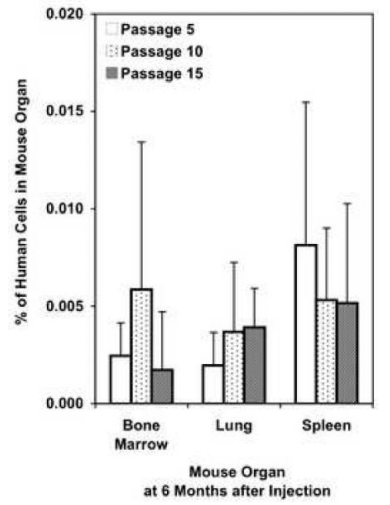

Fig 4. Expansion capacity of hBOEC of different ages

$8 \times 10^{5} \mathrm{hBOEC} / \mathrm{mouse} / \mathrm{day}$ were tail-vein injected into NOD/SCID mice on 3 consecutive days. hBOEC were from passage 5,10 or 15 at the time of injection. Organs were harvested 6 months after injection ( $n=10$ per group). Error bars represent S.D. One experiment. Data represents \% hBOEC per total cells per organ. 

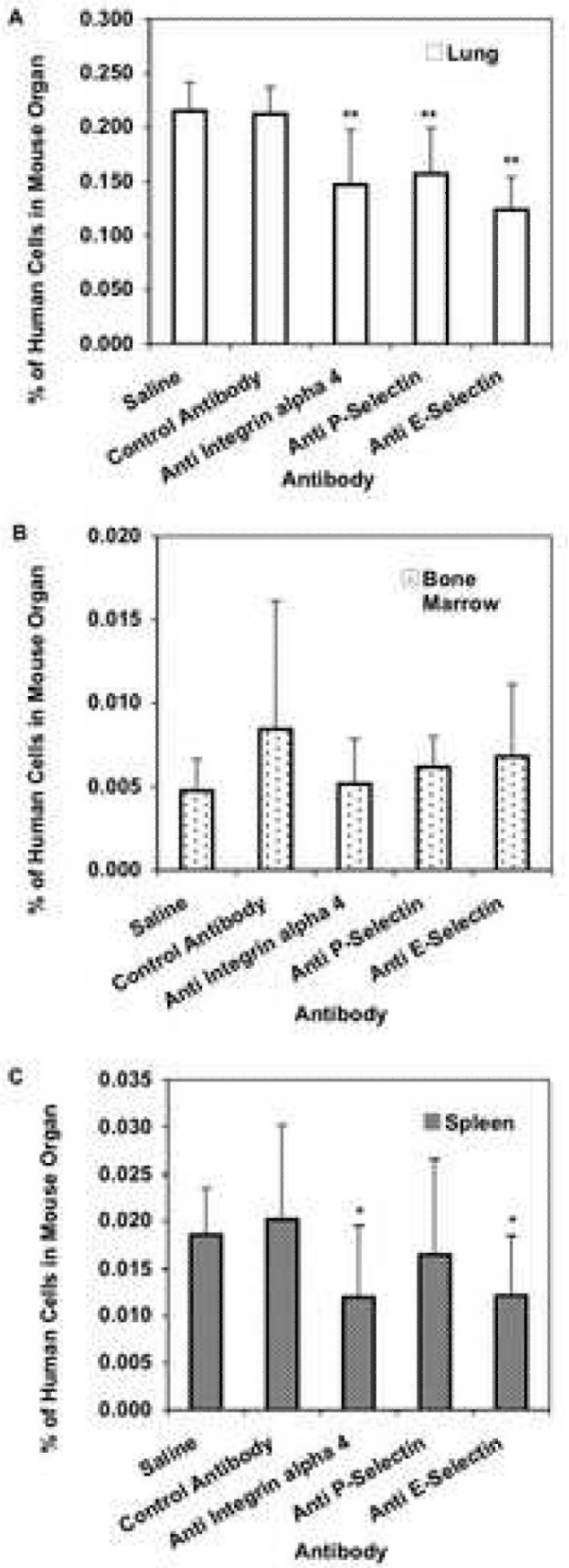

Fig 5. Effect of treating mice with antibodies prior to hBOEC injection

NOD/SCID mice were tail-vein injected with $50 \mu$ grams anti- $\alpha 4$ integrin (Clone R1-2), antiP-selectin (Clone RB40.34) and anti-E-selectin (Clone 10E 9.6) rat anti-mouse antibodies, normal rat IgG or with saline, $1 \mathrm{~h}$ prior to injection of $8 \times 10^{5} \mathrm{BOEC} / \mathrm{mouse}$. Organs were harvested 3 hours later (8 mice per group). Error bars represent S.D. 5A) Lung: ** $\mathrm{P}<0.01$ for each test antibody compared to saline or control antibody. 5B) Bone Marrow: no significant changes. 5C) Spleen: anti- $\alpha 4$ integrin and anti E-selectin had $* \mathrm{P}<0.1$ versus controls. Data confirmed by second experiment (not shown). 

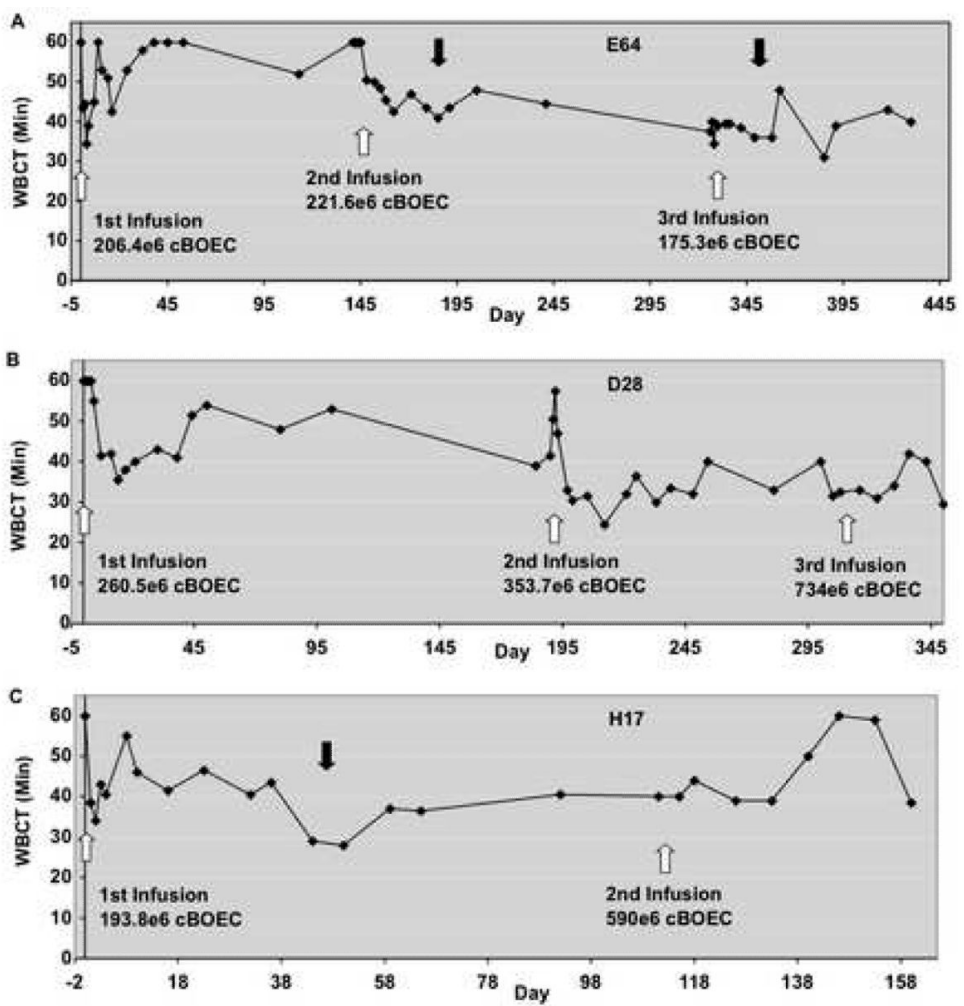

Fig 6. Whole blood clotting time following infusion of cBOEC/FVIII cells Autologous cBOEC/FVIII cells were infused into three hemophilia A dogs: (A) E64, (B) D28 and (C) H17. One or two infusions (depending on cell amount) were given for 3 consecutive days to each dog. This was repeated several months later. Dog H17 received two sets of infusions, whereas E64 and D28 each received three sets. White arrows show infusion date and total number of cells infused. Clotting times were measured for a maximum of $60 \mathrm{~min}$. Pre-infusion WBCT were $>60$ for all 3 dogs. Dark arrows indicate treatment for acute bleeds after altercations. There are 46 time points for E64, 40 for D28 and 24 for $\mathrm{H} 17$. 